\title{
Loss of Wolbachia infection during colonisation in the invasive Argentine ant Linepithema humile
}

\author{
M Reuter ${ }^{1,2}$, JS Pedersen ${ }^{1,3}$ and L Keller ${ }^{1}$ \\ ${ }^{1}$ Department of Ecology and Evolution, University of Lausanne, Bâtiment de Biologie, Lausanne CH-1015, Switzerland; ${ }^{2}$ Department of \\ Biology, University of Oulu, Box 3000, 90014 University of Oulu, Finland; ' ${ }^{3}$ Department of Population Biology, Institute of Biology, \\ University of Copenhagen, Universitetsparken 15, Copenhagen DK-2100, Denmark
}

\begin{abstract}
Wolbachia are maternally inherited bacteria, which are very common in arthropods and nematodes. Wolbachia infection may affect host reproduction through feminisation, parthenogenesis, male-killing, cytoplasmic incompatibility and increased fecundity. Previous studies showing discrepancies between the phylogenies of Wolbachia and its arthropod hosts indicate that infection is frequently lost, but the causes of symbiont extinction have so far remained elusive. Here, we report data showing that colonisation of new habitats is a possible mechanism leading to the loss of infection. The presence and prevalence of Wolbachia were studied in three native and eight introduced populations of the Argentine ant Linepithema humile. The screening shows that the symbiont is common in the three native $L$. humile populations
\end{abstract}

analysed. In contrast, Wolbachia was detected in only one of the introduced populations. The loss of infection associated with colonisation of new habitats may result from drift (founder effect) or altered selection pressures in the new habitat. Furthermore, a molecular phylogeny based on sequences of the Wolbachia wsp gene indicates that $L$. humile has been infected by a single strain. Horizontal transmission of the symbiont may be important in ants as suggested by the sequence similarity of strains in the three genera Linepithema, Acromyrmex, and Solenopsis native from South and Central America.

Heredity (2005) 94, 364-369. doi:10.1038/sj.hdy.6800601

Published online 22 December 2004

Keywords: social insects; Wolbachia; Linepithema; invasive species

\section{Introduction}

Wolbachia are maternally inherited bacterial symbionts of arthropods and nematodes (Werren, 1997; Stouthamer et al, 1999). They are extraordinarily successful, being present in a large number of host species and attaining high prevalence within infected host populations. The spread of Wolbachia through the host population relies on selfish alterations imposed on the host's reproductive physiology. As Wolbachia is transmitted only by females, the symbiont's manipulation acts to increase the number of infected daughters produced by an infected female above the average production of daughters per female (Werren and O'Neill, 1997). This is achieved in one of two ways. Some Wolbachia strains increase the proportion of females among the surviving offspring of infected females by transforming males into functional daughters (feminisation), inducing parthenogenetic reproduction, or provoking the selective abortion of sons (male-killing) in species with sib competition. Alternatively, other strains reduce the reproductive success of uninfected females by a mechanism called cytoplasmic incompatibility (CI), which causes partial or complete infertility of matings between infected males and uninfected females (Rousset and Raymond, 1991; Werren, 1997).

Despite the capacity of Wolbachia to spread rapidly, the lifespan of infection within host populations appears to

Correspondence: $M$ Reuter, The Galton Laboratory, Department of Biology, University College London, 4 Stephenson Way, London NWI 2HE, UK. E-mail:m.reuter@ucl.ac.uk

Received 3 February 2003; accepted 1 July 2004; published online 22 December 2004 be limited. This is suggested by strong discrepancies between the phylogenies of hosts and symbionts, indicating that the distribution of Wolbachia strains among host species is determined by frequent horizontal transmission and loss of infection rather than cospeciation between host and symbiont (Werren et al, 1995; van Meer et al, 1999). Contrary to the mechanisms promoting infection, the factors causing the extinction of Wolbachia within host populations are not well understood (Werren and Windsor, 2000). It has been suggested that infection might be lost when hosts colonise new distant habitats. Thus, native (Argentinean) populations of the fire ant Solenopsis invicta are commonly infected with Wolbachia, while introduced populations, established in the US in the early 20th century, lack the symbiont (Shoemaker et al, 2000). It is not yet clear why the introduction of S. invicta has been associated with a loss of Wolbachia infection. The loss could have been due to a founder event, with all founding individuals being uninfected. Alternatively, the infection could have been lost in the early growth phase of the introduced population, either by drift (small population size) or selection (different environment or population dynamics compared to the source population) (Shoemaker et al, 2000).

In order to assess the general significance of longdistance dispersal as a cause of Wolbachia loss, it is helpful to investigate other species that have been introduced into new habitats. An ideal system is the invasive Argentine ant Linepithema humile, a species known to carry Wolbachia (Jeyaprakash and Hoy, 2000). Like S. invicta, L. humile originates from South America. During the last century, it has been introduced 
worldwide (Passera, 1994). As for many other ant species (Ross and Keller, 1995), introduction events probably have been associated with a major shift in social organisation. Native populations of L. humile are multicolonial and characterised by low densities and high levels of aggression between workers of different nests (Suarez et al, 1999). In contrast, introduced populations are ecologically dominant and unicolonial (Passera, 1994). Nests are socially connected in a dense network throughout the population, resulting in a complete lack of kin structure among nestmate workers and no aggression between workers of different nests of the same 'supercolony', even when these are separated by thousands of kilometres (Giraud et al, 2002).

To test the hypothesis that long-distance dispersal and colonisation might be associated with a loss of infection, we analysed L. humile samples from native populations in Argentina as well as introduced populations in Argentina, Chile, USA, Australia, Spain, and France. Furthermore, we isolated and sequenced the wsp gene to conduct a phylogenetic analysis that included Wolbachia strains isolated from other insects.

\section{Materials and methods}

\section{Sampling}

In this study, we used samples of L. humile workers collected in three native populations in Argentina as well as eight introduced populations. Two of these introduced populations are situated in Argentina, one in Chile, two in the US, one in Australia, one in Spain (Catalonian supercolony), and one in France (European supercolony). Samples were collected between 1997 and 2000 and DNA was extracted from specimens using standard protocols. All DNA extractions included in this study were known to contain DNA because they had been successfully used for analyses of ant nuclear markers. Information on the location of populations and sample sizes are given in Table 1.

\section{Population screening}

All populations were screened for the presence of Wolbachia. For each population, we analysed 20 workers sampled from as many different nests as possible (Table 1). This sample size is sufficient to detect infection (sample of at least one infected individual) with a probability of $95 \%$ even if infection frequency is as low as 0.15 (assuming a binomial distribution). In one population (Tucumán) only a limited number of individuals were available resulting in a smaller sample size.

The presence of Wolbachia in individual workers was detected by PCR amplification of the Wolbachia gene wsp. We used primers wsp $81 \mathrm{~F}$ and wsp691R (Zhou et al, 1998) with PCR conditions as proposed by the authors. Positive and negative controls were included in every PCR reaction. PCR products were run on $1.5 \%$ agarose gels, which were stained with SYBR Gold (Molecular Probes) before photographing.

The association between Wolbachia infection and the population type (native or introduced) was analysed using generalised linear models (GLM). Given the binary nature of the data (presence/absence of infection), we used a binomial error structure (logit link function) and $\chi^{2}$ tests of significance. We first performed a global analysis on the infection status (infected/uninfected) of all individual worker samples as a function of the independent variables 'population type' (native or introduced) and 'population nested in population type'. This analysis suffers from two potential problems. First, populations are represented by variable numbers of nests and variable numbers of individuals per nest. Second, some introduced populations might be derived from a single and same event of introduction and hence do not represent independent samples. We therefore repeated the analysis with a minimal data set, by using nests and not individuals as sampling units (scoring a nest as infected if at least one worker had been found positive) and including only those introduced populations that differ in the composition of ant mitochondrial genotypes and thus most probably represent independent events of introduction (V Vogel, JS Pedersen, L Keller, unpublished data). The populations included in the minimal data set were Tucumán, Corrientes, Buenos Aires, La Rioja 2, Chile, Hawaii, and Catalonia.

\section{Local prevalence}

To estimate the prevalence of Wolbachia in infected populations, we undertook a more detailed screening in the populations Corrientes and Buenos Aires. We analysed 10 workers from each of 23 (Corrientes) and 26 (Buenos Aires) nests. A GLM was used to detect differences in prevalence between populations and nests. The analysis included both populations, with the infection status of individual workers as dependent variable and the factors 'population' and 'nest nested within population' as independent variables. All statistical tests were performed with the statistical package $\mathrm{R}$ (Ihaka and Gentleman, 1996).

\section{Sequencing}

We sequenced the Wolbachia gene wsp from a small number of infected individuals to determine the identity of the symbiont strains found in the different populations. The sample included three individuals from Buenos Aires and one from each of the populations in Corrientes, Hawaii, and Tucumán. The wsp gene was amplified from these samples using the same methods as for screening. Amplified DNA was recovered from excised gel slices and cloned with the help of a TOPO TA cloning kit (Invitrogen). Plasmids were extracted with the Wizard Plus SV Minipreps DNA Purification System (Promega) and sequenced using the ABI BigDye Terminator Cycle Sequencing Kit (Applied Biosystems). For each host ant, one clone was sequenced on both strands. The forward and reverse sequences from same clones were aligned and corrected by hand to obtain an unambiguous sequence for each clone. A consensus sequence has been deposited on Genbank under accession number AF546756.

\section{Phylogenetic analysis}

A phylogenetic analysis was performed to assess the position of the Wolbachia detected in this study within the Wolbachia A-group as well as their phylogenetic relationship with Wolbachia strains detected in other ant species. The sequence matrix included the wsp sequences of the Wolbachia reference strains defining phylogenetic clades within the Wolbachia phylogeny (van Meer et al, 1999): 
wMel (AF020063), wAlbA (AF020058), wMors (AF020079), wKue (AF071911), wRi (AF020070), wUni (AF020071), wHa (AF020073), wPap (AF020082), wAus (AF020077), and wDro (AF071910). Furthermore, we included wsp sequences from all A-group Wolbachia detected so far in ants, that is, S. invicta A (AF243435, Shoemaker et al, 2000), S. richteri (AF243435, Shoemaker et al, 2000), Acromyrmex octospinosus A1 (AF472557, van Borm et al, 2003), A. echinatior A1 (AF472558, van Borm et al, 2003), A. insinuator A1 (AF47255, van Borm et al, 2003), Formica exsecta wFex2 (AY101197, Reuter and Keller, 2003), and F. exsecta wFex4 (AY101199, Reuter and Keller, 2003, identical to strain AF472557 from F. truncorum). Three additional Wolbachia strains from F. exsecta, wFex1, wFex3, and wFex5, were not included because their ws $p$ genes are most likely recombinants (Reuter and Keller, 2003). The wsp sequence of the B-group Wolbachia strain wCon (AF020083, van Meer et al, 1999) was included in order to root the tree. Sequences were aligned with the help of ClustalX (Thompson et al, 1997) and alignments corrected by hand. Two regions (positions 73-99 and 214-249) could not be aligned with certainty and were excluded from the data set. This resulted in a final alignment of 450 nucleotides in length. Identical sequences were grouped into one taxon. A phylogenetic tree was constructed in PAUP* $4.0 \mathrm{~b} 10$ (Swofford, 1998) using the neighbour-joining method with the Kimura two-parameter distance measure. Bootstrap analysis was performed with 1000 replicates.

\section{Results}

\section{Population screening}

Individuals infected with Wolbachia were detected in four populations (Table 1). While the three native populations of L. humile (Tucumán, Corrientes, and Buenos Aires) were found to be infected, Wolbachia was detected in only one of the eight introduced populations (Hawaii).

The GLM analyses showed a significant difference in prevalence between native and introduced populations both in the global analysis and the analysis of the minimal data set (Tables $2 a$ and $b$, 'population type'). Within the native and introduced range populations also differed significantly (Tables $2 \mathrm{a}$ and $\mathrm{b}$, 'population nested in population type'). This between-population variance in infection is most likely due to the high prevalence found in the Tucumán population (where a single nest was analysed) compared to Buenos Aires and Corrientes, as well as due to the difference in infection between the population in Hawaii and those in the rest of the introduced range.

\section{Local prevalence}

In the two native populations of L. humile where several nests were analysed, the proportion of individuals infected across all nests were $0.27 \pm 0.16 / 0.3 / 0-0.5$ (mean $\pm \mathrm{SD} /$ median/range) in Corrientes and $0.33 \pm 0.22 / 0.3 / 0-0.8$ in Buenos Aires. The GLM analysis revealed that prevalence did not differ significantly between the two populations, but varied significantly between nests within populations (Table 3 ).

\section{Sequencing and phylogeny}

Within the six sequences of 608 nucleotides, only four sites were variable due to nucleotide substitutions in single sequences. All four nucleotide substitutions were silent (ie do not result in an altered amino-acid

Table 2 Results of GLM analyses of Wolbachia infection of individual worker samples in native and introduced populations of L. humile. (a) Global analysis of all worker samples. (b) Analysis of the minimal data set (see Materials and methods section for details)

\begin{tabular}{lccc}
\hline Factor & $d f$ & P-value & $\begin{array}{c}\text { Explained D } \\
(\%)\end{array}$ \\
\hline (a) Global analysis & & & \\
$\quad$ Population type & 1 & $<0.0001$ & 40.3 \\
$\quad$ Population within population type & 9 & 0.015 & 13.4 \\
& & & \\
(b) Minimal data set & 1 & 0.0002 & 16.2 \\
$\quad$ Population type & & & \\
$\quad$ Population within population type & 5 & 0.035 & 14.4 \\
\hline
\end{tabular}

The dependent variable was the infection status of workers (infected/uninfected) in the global analysis and the infection status of nests (infected/uninfected) in the analysis of the minimal data set. The table shows the independent variables entered in the analysis (Factor), the number of their degrees of freedom (df), and their significance calculated by a $\chi^{2}$ test ( $P$-value). Further, the percentage of the total deviance explained by the significant factors is given (Explained $D$ ).

Table 1 L. humile samples included in the screening for infection by Wolbachia

\begin{tabular}{|c|c|c|c|c|c|}
\hline Name & Locality & Type & No. of nests & $\begin{array}{c}\text { No. of individuals } \\
\text { in total }\end{array}$ & $\begin{array}{l}\text { No. of positive } \\
\text { individuals (\%) }\end{array}$ \\
\hline Tucumán & Ticucho, Tucumán, Argentina & Native & 1 & 11 & $9(82 \%)$ \\
\hline Corrientes & Arroyo Cuay Grande, Corrientes, Argentina & Native & 20 & 20 & $4(20 \%)$ \\
\hline Buenos Aires & Otamendi, Buenos Aires, Argentina & Native & 20 & 20 & $10(50 \%)$ \\
\hline La Rioja 1 & Chuquis, La Rioja, Argentina & Introduced & 20 & 20 & 0 \\
\hline La Rioja 2 & Aminga, La Rioja, Argentina & Introduced & 14 & 20 & 0 \\
\hline Chile & La Cruz, Santiago, Chile & Introduced & 10 & 20 & 0 \\
\hline California & Davis, California, USA & Introduced & 10 & 20 & 0 \\
\hline Hawaii & Haleakala, Hawaii, USA & Introduced & 10 & 20 & $2(10 \%)^{\mathrm{a}}$ \\
\hline Melbourne & Bundoora, Melbourne, Australia & Introduced & 20 & 20 & 0 \\
\hline Port Leucate & Port Leucate, France & Introduced & 10 & 20 & 0 \\
\hline Catalonia & Catalonia, Spain & Introduced & $4+1+1^{\mathrm{b}}$ & 20 & 0 \\
\hline
\end{tabular}

aFrom two different nests.

${ }^{\mathrm{b} N e s t s} 4,1$, and 1, respectively, were sampled from three different localities within the Catalonian supercolony (Giraud et al, 2002). 
Table 3 Results of the GLM analysis of Wolbachia infection in native populations Buenos Aires and Corrientes

\begin{tabular}{lrcc}
\hline Factor & $d f$ & P-value & Explained D \\
\hline Population & 1 & 0.14 & - \\
Nest within population & 47 & $<0.0001$ & $16.2 \%$ \\
\hline
\end{tabular}

The dependent variable in the analysis was the infection status of workers (infected/uninfected). The table shows the independent variables entered in the analysis (Factor), the number of their degrees of freedom (df), and their significance calculated by a $\chi^{2}$ test $(P$-value). Further, the percentage of the total deviance explained by the significant factors is given (Explained $D$ ).

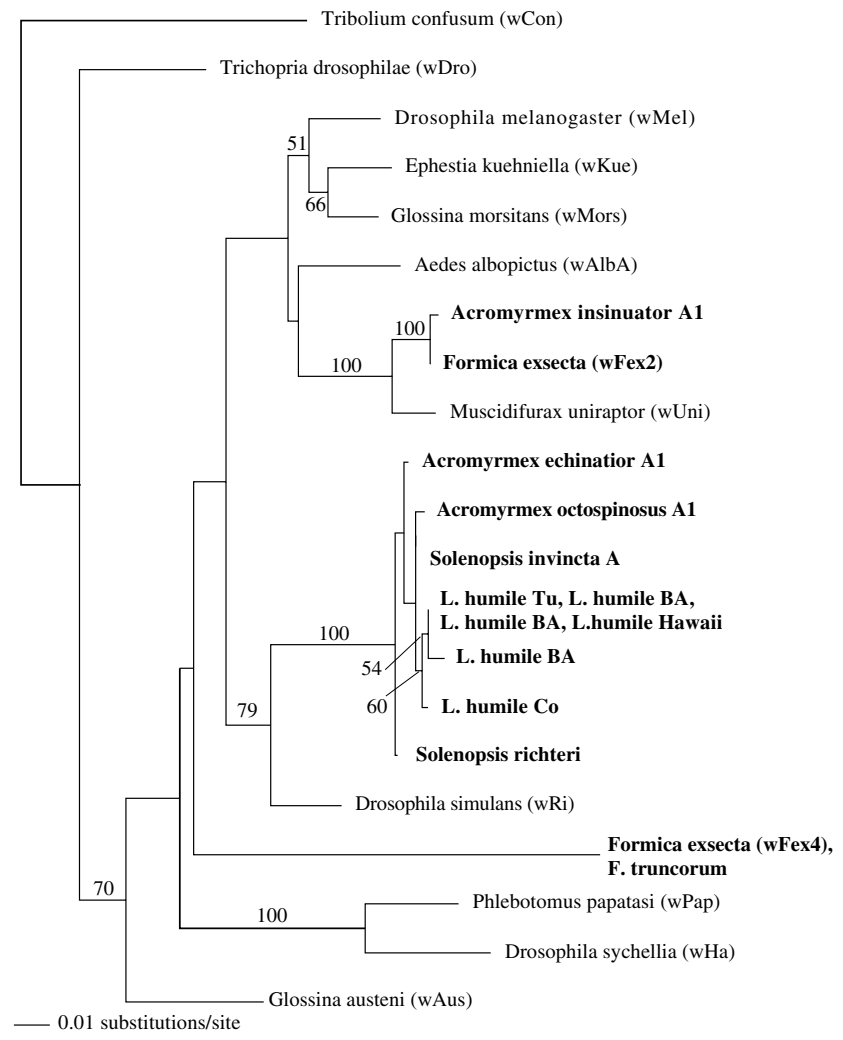

Figure 1 Phylogenetic tree of Wolbachia wsp sequences constructed with neighbour-joining and Kimura two-parameter distances. Wolbachia strains are designated by the name of their host species or strain names. Sequences from ant hosts are marked by bold face. $\mathrm{Tu}, \mathrm{BA}$, and Co indicate L. humile from populations in Tucumán, Buenos Aires, and Corrientes. Numbers next to nodes indicate the statistical support as the percentage of 1000 bootstrap replicates in which the taxa of a group were placed together in a monophyletic clade.

sequence), but it must be noted that the degree of variation observed is in the range of Taq polymerase error (Sanson et al, 2002). Owing to the similarity in wsp sequences, the Wolbachia lineages from L. humile form a monophyletic group in the wsp phylogeny (Figure 1). The phylogeny also reveals that the Wolbachia from L. humile cluster together with the symbiont lineages identified in other ant species from South and Central America, $A$. echinatior, A. octospinosus, S. invicta, and S. richteri. The eight Wolbachia lineages are genetically very similar and form a very strongly supported monophyletic group (Figure 1).

\section{Discussion}

Our study shows that the level of Wolbachia infection in the Argentine ant L. humile differs greatly between native and introduced populations. Whereas Wolbachia is common in ants from native South American populations, only one of the eight introduced populations is infected. This pattern of infection is identical to that found in the fire ant S. invicta (Shoemaker et al, 2000).

There are several possible mechanisms that could lead to the observed pattern of infection (Shoemaker et al, 2000). First, the difference in infection between the two population types could be independent of introduction events and arise because (i) native populations have been infected after the introduction into new habitats, (ii) infection occurs in introduced populations but remained undetected, and (iii) introduced populations originate from an uninfected native population not included in our sample. All three of these hypotheses are unlikely to apply. Since the three native populations analysed are separated by long distances $(650-1050 \mathrm{~km})$ covering most of the species' native range and because Argentine ants have been introduced worldwide less than 100 years ago, it is doubtful that Wolbachia would have spread in native populations only after the establishment of introduced populations. It is also unlikely that we failed to detect Wolbachia in introduced populations. Our screening provided a high probability of detecting Wolbachia infections even at relatively low prevalence, and certainly at a prevalence comparable to that observed in native populations (30\%): with a sample size of 20 ants (as used in the study), the detection probability is higher than 0.999. Finally, it is improbable that the foundresses of all uninfected introduced populations stem from an uninfected native population. The invasive populations of L. humile outside South America most likely originate from the Buenos Aires region (V Vogel, JS Pedersen, L Keller, unpublished data; Tsutsui et al, 2001), which was found to be infected in the present study.

Given the absence of a convincing alternative explanation, it seems most likely that the loss of Wolbachia infection is associated with the introduction of the ants into novel habitats. This conclusion is further corroborated by the difference in infection rate between the introduced populations from the US, Australia, and Europe and its putative source, the native population of Buenos Aires. The loss of Wolbachia infection during introduction could have occurred via one of three processes. First, Wolbachia could have been eliminated through drift during introduction if all founder queens were uninfected. Although possible, this scenario is highly unlikely. European populations of L. humile originate from at least six to 12 foundress queens (Giraud et al, 2002). Assuming that the prevalence in native populations is $30 \%$, the probability that all foundresses of introduced populations were uninfected ranges from $1.4 \%$ (12 foundresses) to $12 \%$ (six foundresses). These estimates decrease considerably if we take into account the fact that there have probably been several independent events of introduction (Tsutsui et al, 2001; Giraud et al, 2002). With only two events of introduction of six or 12 queens each, the probability of all foundresses being uninfected drops to $1.4 \%$ (six foundresses per introduction) and $0.02 \%$ (12 foundresses). Alternatively, Wolbachia may have been lost by drift after the introduction into 
new habitats. This would be particularly likely if Wolbachia caused CI. CI Wolbachia are likely to be lost by selection if their frequency drops below a threshold frequency determined by the rate of vertical transmission and the effect of infection on host fertility (Caspari and Watson, 1959; Hoffmann and Turelli, 1997). Strong drift during and after introduction might have reduced infection frequency in novel populations below this threshold and thus have lead to a loss of the symbiont. Finally, Wolbachia might have been lost in introduced populations because infection is counter-selected in the new habitat, even at frequencies that represent a stable equilibrium in native conditions. It is possible that the parameters governing infection dynamics (eg the rate of horizontal transmission and the impact of infection on host fitness) differ between native and introduced population because of environmental and ecological differences. Of interest in this context is the fact that introduced populations have a different social structure than native populations. In particular, introduced populations have gone through a prolonged phase of exponential growth and the social structure has changed from multicoloniality to unicoloniality with the population forming a dense network of interconnected nests (see Introduction). Such changes may play a significant role in the dynamics of propagation of Wolbachia and be involved in the loss of infection in introduced populations.

Our analysis of the Wolbachia gene wsp revealed strong sequence similarity between the genes from bacteria found in different populations of L. humile and in four other ant species from South and Central America, $A$. octospinosus, A. echinatior, S. invicta, and $S$. richteri (Figure 1). The observed sequence similarity can have different causes. The first is that hosts have inherited their Wolbachia from a common ancestor carrying an infection. This is a likely explanation for the similarity between Wolbachia sequences of different L. humile populations. Second, sequence similarity can arise due to horizontal transmission of bacteria between hosts. Horizontal transmission could explain why closely related Wolbachia occur in the distantly related ant genera Acromyrmex, Solenopsis, and Linepithema. The vector of horizontal transmission could have been parasites or parasitoids specialised on ants (van Borm et al, 2003). Finally, it should be noted that sequence similarity might also stem from homologous recombination between Wolbachia lineages. Recombinant Wolbachia strains have been recently described (Werren and Bartos, 2001; Reuter and Keller, 2003), showing that genetic exchange is possible between intracellular symbionts. In order to determine whether recombination has played a role in generating the sequence similarity described here, more genes should be sequenced from Wolbachia in Acromyrmex, Solenopsis, and Linepithema. This would allow to determine whether sequence similarity is restricted to certain regions of the Wolbachia genome, as predicted if recombination has taken place.

In conclusion, this study demonstrates that the introduction of the Argentine ant has been frequently accompanied by the loss of Wolbachia infection. The most likely explanation is that the loss resulted from a change in the parameters governing infection dynamics possibly because of environmental differences between the native and introduced range and/or a shift in social organisa- tion that was associated with the introduction. Whatever the causes responsible for the loss of Wolbachia in the introduced range, this study indicates that long-distance dispersal and colonisation of new habitats can affect the dynamics of infection by Wolbachia and ultimately cause the loss of infection. Finally, the finding that several ant genera of South and Central American harbour very similar stains of Wolbachia indicates that horizontal transmission of Wolbachia may be common in ants.

\section{Acknowledgements}

We thank Juan Briano, Luis Calcaterra, Maria Chiotis, Ross Crozier, Tatiana Giraud, Michael Krieger, Paul Krushelnycky, Renato Ripa, and Philip S Ward for providing ant samples. Nadia Bruyndockx and Marion Tanner are gratefully acknowledged for their help in the laboratory. Karen Parker kindly agreed to check our English. This study was supported by grants from the Swiss National Science Foundation, the Carlsberg Foundation, the Swiss Academy of Sciences, and the European Community's Improving Human Potential Programme under Contract HPRN-CT-2000-00052 'INSECTS'.

\section{References}

Caspari E, Watson GS (1959). On the evolutionary importance of cytoplasmic sterility in mosquitoes. Evolution 13: 568-570.

Giraud T, Pedersen JS, Keller L (2002). Evolution of supercolonies: the Argentine ants of southern Europe. Proc Natl Acad Sci USA 99: 6075-6079.

Hoffmann AA, Turelli M (1997). Cytoplasmic incompatibility in insects. In: O'Neill SL, Hoffmann AA, Werren JH (eds) Influential Passengers. Oxford University Press: Oxford. pp 42-80.

Ihaka R, Gentleman R (1996). R: a language for data analysis and graphics. J Comput Graph Stat 5: 299-314.

Jeyaprakash A, Hoy MA (2000). Long PCR improves Wolbachia DNA amplification: wsp sequences found in $76 \%$ of sixtythree arthropod species. Insect Mol Biol 9: 393-405.

Passera L (1994). Characteristics of tramp species. In: Williams DF (ed) Exotic Ants, Biology, Impact, and Control of Introduced Species. Westview Press: Boulder. pp 23-43.

Reuter M, Keller L (2003). High levels of multiple infection and recombination of Wolbachia in the ant Formica exsecta. Mol Biol Evol 20: 748-753.

Ross KG, Keller L (1995). Ecology and evolution of social organization - insights from fire ants and other highly eusocial insects. Annu Rev Ecol Syst 26: 631-656.

Rousset F, Raymond M (1991). Cytoplasmic incompatibility in insects: why sterilize females. Trends Ecol Evol 6: 54-57.

Sanson GFO, Kawashita SY, Brunstein A, Briones MRS (2002). Experimental Phylogeny of neutrally evolving DNA sequences generated by a bifurcate series of nested polymerase chain reactions. Mol Biol Evol 19: 170-178.

Shoemaker DD, Ross KG, Keller L, Vargo EL, Werren JH (2000). Wolbachia infections in native and introduced populations of fire ants (Solenopsis spp.). Insect Mol Biol 9: 661-673.

Stouthamer R, Breeuwer JAJ, Hurst GDD (1999). Wolbachia pipientis: microbial manipulator of arthropod reproduction. Ann Rev Microbiol 53: 71-102.

Suarez AV, Tsutsui ND, Holway DA, Case TJ (1999). Behavioral and genetic differentiation between native and introduced populations of the Argentine ant. Biol Invasions 1: 43-53.

Swofford DL (1998). PAUP*. Sinauer Associates: Sunderland, MA.

Thompson JD, Gibson TJ, Plewniak F, Jeanmougin F, Higgins DG (1997). The ClustalX windows interface: flexible strategies 
for multiple sequence alignment aided by quality analysis tools. Nucleic Acids Res 25: 4876-4882.

Tsutsui ND, Suarez AV, Holway DA, Case TJ (2001). Relationships among native and introduced populations of the Argentine ant (Linepithema humile) and the source of introduced populations. Mol Ecol 10: 2151-2161.

van Borm S, Wenseleers T, Billen J, Boomsma JJ (2003). Cloning and sequencing of wsp encoding gene fragments reveals a diversity of co-infecting Wolbachia strains in Acrmyrmex leafcutter ants. Mol Phylogenet Evol 26: 102-109.

van Meer MMM, Witteveldt J, Stouthamer R (1999). Phylogeny of the arthropod endosymbiont Wolbachia based on the wsp gene. Insect Mol Biol 8: 399-408.

Werren JH (1997). Biology of Wolbachia. Ann Rev Entomol 42: 587-609.
Werren JH, Bartos JD (2001). Recombination in Wolbachia. Curr Biol 11: 431-435.

Werren JH, O'Neill SL (1997). The evolution of heritable symbionts. In: O'Neill SL, Hoffmann AA, Werren JH (eds) Influential Passengers. Oxford University Press: Oxford. pp 1-41.

Werren JH, Windsor DM (2000). Wolbachia infection frequencies in insects: evidence of a global equilibrium? Proc $R$ Soc Lond Ser B 267: 1277-1285.

Werren JH, Zhang W, Guo LR (1995). Evolution and phylogeny of Wolbachia: reproductive parasites of arthropods. Proc $R$ Soc Lond Ser B 261: 55-63.

Zhou WG, Rousset F, O'Neill S (1998). Phylogeny and PCRbased classification of Wolbachia strains using wsp gene sequences. Proc R Soc Lond Ser B 265: 509-515. 